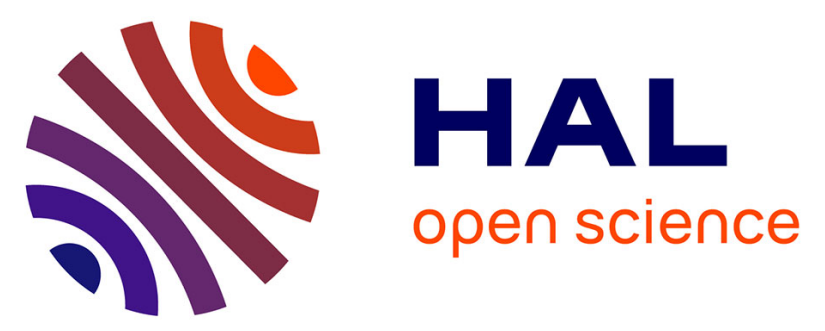

\title{
Numerical study and theoretical modelling of void growth in porous ductile materials subjected to cyclic loadings
}

Rémi Lacroix, Jean-Baptiste Leblond, Gilles Perrin

\section{- To cite this version:}

Rémi Lacroix, Jean-Baptiste Leblond, Gilles Perrin. Numerical study and theoretical modelling of void growth in porous ductile materials subjected to cyclic loadings. European Journal of Mechanics - A/Solids, 2016, 55, pp.100-109. 10.1016/j.euromechsol.2015.08.010 . hal-01191778

\section{HAL Id: hal-01191778 \\ https://hal.sorbonne-universite.fr/hal-01191778}

Submitted on 2 Sep 2015

HAL is a multi-disciplinary open access archive for the deposit and dissemination of scientific research documents, whether they are published or not. The documents may come from teaching and research institutions in France or abroad, or from public or private research centers.
L'archive ouverte pluridisciplinaire HAL, est destinée au dépôt et à la diffusion de documents scientifiques de niveau recherche, publiés ou non, émanant des établissements d'enseignement et de recherche français ou étrangers, des laboratoires publics ou privés. 


\title{
Numerical study and theoretical modelling of void growth in porous ductile materials subjected to cyclic loadings
}

\author{
Rémi Lacroix ${ }^{1}$, Jean-Baptiste Leblond ${ }^{2}$, Gilles Perrin $^{3}$ \\ ${ }^{1}$ ESI-France, Le Récamier, 70 rue Robert, 69006 Lyon, France \\ ${ }^{2}$ UPMC Univ Paris 06, UMR 7190, Institut Jean Le Rond d'Alembert, Tour 65-55, 4 place \\ Jussieu, 75005 Paris, France; tel.33144273924, e-mail jbl@lmm.jussieu.fr \\ ${ }^{3}$ AREVA, Tour Areva, 1 place Jean Millier, 92084 Paris La Défense Cedex, France
}

\begin{abstract}
Experiments have shown that in porous ductile materials, cyclic loadings lead to lower fracture strains than monotone ones. The effect has been tentatively ascribed to a continued increase of the mean porosity during each cycle with the number of cycles ("ratcheting of the porosity"). In this work, we first perform finite-element-based micromechanical simulations of elementary hollow cells. These cells are initially spherical, contain an initially spherical void and are loaded cyclically through conditions of homogeneous boundary strain rate; the triaxiality is held constant throughout in absolute value. These simulations fully confirm the interpretation of the reduced fracture strains under cyclic loadings just mentioned. The modelling of the ratcheting of the porosity is then discussed. Gurson (1977)'s classical model is shown not to be able to predict such an effect, the evolution of the porosity being stabilized right from the first semicycle. The so-called LPD model due to Leblond et al. (1995), an improved variant of Gurson (1977)'s model with a more refined description of strain hardening, makes a better job but fails to accurately reproduce the results of the micromechanical simulations. One explanation of the discrepancy is the assumption of positively proportional straining made in this model, which is basically inadequate for cyclic loadings. An improved version of the LPD model is introduced; this version discards this assumption, at the expense of introduction and radial discretization of an underlying spherical "microcell" at each material point. It is not significantly more computationally expensive than the old one and permits a satisfactory reproduction of the results of the micromechanical simulations. This paves the way to simulations of ductile rupture under cyclic loadings within the framework of Gurson-like models.
\end{abstract}

Key words: Porous ductile materials, cyclic loadings, ratcheting of the porosity, numerical simulations, theoretical modelling

* Corresponding author. 


\section{Introduction}

Experiments performed on both CT specimens (Kobayashi et al., 1991) and cracked pipes (Schmidt et al., 1991) have clearly shown that in ductile metals, the strain to fracture is considerably lower, for a given load level, if this load is reached under cyclic conditions than if it is reached monotonically. The experiments depicted in Schmidt et al. (1991)'s report emphasize the influence of strain hardening upon this reduction of ductility: it is more important for stainless steels, exhibiting considerable strain hardening, than for less hardenable low-alloy steels.

The problem was investigated from a more theoretical point of view by Gilles et al. (1992). After due elimination of other possible explanations, they concluded that the reduction of ductility under cyclic loadings probably arises from an effect of gradual increase of the mean porosity during one cycle with the number of cycles. This effect will be termed ratcheting of the porosity in the sequel, by analogy with the more usual ratcheting of the strain. To support their conclusion, Gilles et al. (1992) performed finite-element based micromechanical simulations of cylindrical elementary cells made of some hardenable von Mises material. These cells contained an initially spherical void and were subjected to cyclic loadings under conditions of fixed triaxiality in absolute value. The simulations confirmed the existence of the ratcheting of the porosity anticipated by the authors. This finding was however somewhat impaired by the fact that the simulations involved fluctuations of the triaxiality of the order of a percent. Since the porosity rate is highly sensitive to the value of the triaxiality (as was shown in numerous works starting with the seminal one of Rice and Tracey (1969)), such fluctuations might be sufficient to explain a small increase of the mean porosity from one cycle to the next one.

Gilles et al. (1992)'s micromechanical simulations and analysis were improved by Devaux et al. (1997). First, these authors performed simulations analogous to those of Gilles et al. (1992) but with a much better control of the triaxiality, thus eliminating any possible doubts on the accuracy of the results. The new simulations confirmed the existence of the ratcheting of the porosity in a definitive way. Second, they showed that Gurson (1977)'s classical model for porous ductile materials does not predict this phenomenon, but stabilization of the evolution of the porosity right from the first semi-cycle. This wrong prediction was shown to arise from a too crude modelling of strain hardening within the model. Its weak point was shown to be the fact that the same "average yield stress of the matrix" appears in both the "square" and "cosh" terms of Gurson's yield function. Finally Devaux et al. (1997) compared the results of their simulations to the predictions of the so-called LPD model (Leblond et al., 1995). This is a variant of Gurson (1977)'s model with an improved description of strain hardening, involving distinct "average yield stresses of the matrix" in the "square" and "cosh" terms of the yield function. It was concluded that the predictions of the LPD model for cyclic loadings are qualitatively better than those of Gurson's model, but quantitatively still liable to improvement.

In spite of the points it made, Devaux et al. (1997)'s conference proceedings paper was not deemed conclusive enough by the authors to warrant publication in a scientific journal. Its main drawback was judged to be its lack of proposal of a Gurson-like model able to accurately reproduce the results of micromechanical simulations of the ratcheting of 
the porosity. Such a model is indispensable for future finite-element based simulations of ductile rupture of full-size specimens or structures under cyclic loadings.

Later papers on the subject included the works of Besson and Guillemer-Neel (2003), Brocks and Steglich (2003), Rabold and Kuna (2005), Steglich et al. (2005) and Mbiakop et al. (2014). They were essentially devoted to further micromechanical simulations along the lines initiated by Gilles et al. (1992) and Devaux et al. (1997), sometimes for more complex (cubical) elementary cells. An effort was made in most papers to compare the results of the new simulations to various model predictions. However the match was not generally better than that found by Devaux et al. (1997) for the LPD model, and no new, more satisfactory model was proposed. Thus the conclusions reached merely confirmed those of Devaux et al. (1997).

It is obvious from the description of these studies that they still leave considerable room for improvement. Among other things:

- If the goal is to define a new Gurson-type model better fit for cyclic loadings, it is preferable to perform micromechanical simulations for spherical elementary cells, rather than cylindrical ones. Indeed such models are based on approximate homogenization of the former type of cell. ${ }^{1}$

- Similarly, micromechanical simulations with artificially enhanced values of Young's modulus are desirable. Indeed the homogenization procedure underlying the derivation of Gurson-type models is always based on neglect of elasticity.

- More importantly, the mitigated success of the LPD model evidenced by Devaux et al. (1997), in the case of cyclic loadings, essentially arose from the hypothesis made in this model of positively proportional straining of the elementary cell considered. This simplifying assumption is completely unrealistic for cyclic loadings. It was introduced in order to get an analytic expression of the distribution of strain hardening within the cell resulting from the previous mechanical history. The development of computer storage capabilities permits to now drop this unrealistic hypothesis and analytical calculation. This may be done at the expense of numerical calculation and storage of a large but still reasonable number of internal variables.

The aim of this paper is to improve the numerical study and the theoretical modelling of the ratcheting of the porosity under cyclic loadings along these lines. The paper is organized as follows:

- Section 2 presents some new micromechanical simulations of an elementary porous cell subjected to cyclic loadings. These simulations differ from previous ones essentially through the shape of the cell (spherical instead of cylindrical or cubical) and the very high value of Young's modulus.

- Section 3 recalls Devaux et al. (1997)'s proof of absence of ratcheting of the porosity under cyclic loadings within Gurson (1977)'s original model. This proof is provided for

$\overline{1}$ Of course, spherical cells are less easily "stacked up" than cylindrical ones to build a fully dense material, and in this sense less "realistic". But they are nevertheless preferable in a first step for the sake of accurate assessment of the model; later adjustment to other cell shapes is always possible for instance through introduction of Tvergaard (1981)'s famous heuristic $q$-parameter. 
completeness and because Devaux et al. (1997)'s work is not easily accessible.

- Section 4 briefly expounds the equations of the LPD model in its original version (Leblond et al., 1995). A minimum but indispensable presentation of the underlying homogenization procedure is included.

- Finally Section 5 presents the improved version of the LPD model proposed, together with a comparison of its predictions with the results of the micromechanical simulations.

\section{Micromechanical simulations of void growth under cyclic loadings}

\subsection{Preliminary remarks}

Before embarking on any micromechanical simulation of elementary hollow cells loaded cyclically, it is useful to make some qualitative remarks. These remarks are independent of any "homogenized" model used to approximately describe the behaviour of such cells.

- In the absence of strain hardening and elasticity, the evolution of the porosity must stabilize instantaneously (that is right from the first semi-cycle) under conditions of proportional overall straining or stressing. To establish such a property, note that if the stress tensor changes sign, the von Mises equivalent stress remains unchanged whereas the plastic strain rate tensor changes sign. (This results from the symmetry of the yield locus with respect to the origin, combined with the normality property of the plastic flow rule). It follows that given some velocity and stress fields satisfying the instantaneous equations of elastoplasticity on a given configuration, one obtains another possible instantaneous solution by simply changing the signs of these fields. The conclusion follows through integration in time during the tensile and compressive phases of each cycle: the full sequence of cell geometries and porosities met during the first tensile or compressive semi-cycle is met in reverse order during the following compressive or tensile semi-cycle. ${ }^{2}$

- Strain hardening destroys the symmetry of the tensile and compressive phases of each cycle. Indeed the continuous increase of the yield stress (for isotropic hardening), or the displacement of the center of the yield surface (for kinematic hardening), introduce some irreversibility. In the case of isotropic hardening for instance, closing a pre-opened void requires a higher stress in absolute value than closing a pristine one, because of the increased yield stress of the surrounding matrix.

- Elasticity also destroys the symmetry of the tensile and compressive phases of each cycle. Indeed it introduces, upon each change of sign of the load, a change of regime from a plastic loading phase to an elastic unloading phase. Thus the beginning of a tensile or compressive semi-cycle is not symmetrical to the end of the preceding compressive or tensile semi-cycle.

$\overline{2}$ This somewhat paradoxical reversibility property for rigid-ideal-plastic solids is the analog, for solid mechanics, of the classical reversibility property of Stokes's equations, for extremely viscous fluids. 
A consequence of what precedes is that the ratcheting of the porosity, if present, is fundamentally tied to two features of the material behaviour: strain hardening and elasticity. With regard to strain hardening, there is a connection here with Schmidt et al. (1991)'s observation that the steeper the hardening curve, the stronger the reduction of the fracture strain in cyclic experiments. On the other hand, the effect of elasticity upon the ratcheting of the porosity, although strictly nonzero, may be considered as minor, because elastic strains are generally much smaller than plastic ones in problems of ductile rupture.

We shall therefore concentrate hereafter on the sole effect of strain hardening by considering rigid-hardenable materials. In addition, hardening will be assumed to be of isotropic type, following the hypothesis made in most theoretical homogenized models and notably that of Gurson (1977). The study of kinematic hardening will be envisaged in some future work.

\subsection{Principle of the simulations}

The micromechanical simulations are quite analogous to those performed by Koplik and Needleman (1988), the initiators of such numerical studies of elementary cells in porous ductile materials, except for the cyclic character of the loadings. An initially spherical cell $\Omega$ of centre $O$, radius $b$, containing an initially concentric spherical void $\omega$ of radius $a$, is subjected to some axisymmetric loading with axis of rotational symmetry $O y$ (Figure 1).

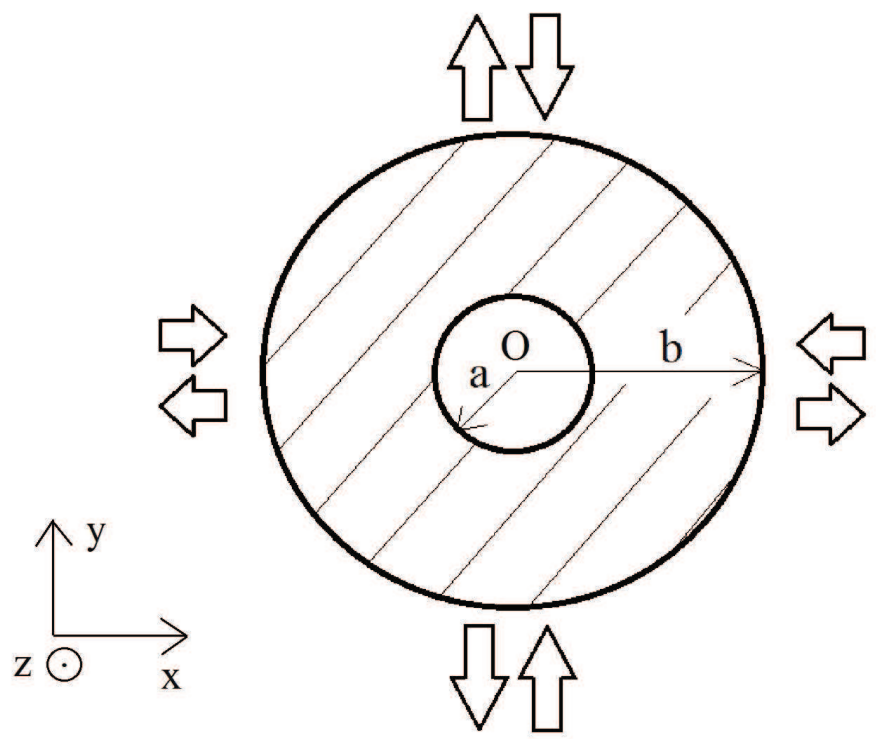

Fig. 1. Schematic view of the cell and its loading

The loading is imposed through conditions of homogeneous boundary strain rate. These conditions, defined by Mandel (1964) and Hill (1967), were used by various authors in the derivation of homogenized, Gurson-type models. They read:

$$
\mathbf{v}(\mathbf{x})=\mathbf{D} \cdot \mathbf{x} \quad \forall \mathbf{x} \in \partial \Omega \quad, \quad D_{x x}=D_{z z} \neq D_{y y} \text {, other } D_{i j}=0,
$$

where $\mathbf{v}$ denotes the velocity, $\mathbf{x}$ the current position, $\mathbf{D}$ the overall (symmetric) strain 
rate tensor, and $\partial \Omega$ the boundary of $\Omega$. The nonzero components $D_{x x}=D_{z z}, D_{y y}$ of $\mathbf{D}$ are continuously adjusted so as to respect conditions of proportional overall stressing:

$$
\Sigma(t)=\lambda(t) \Sigma_{0}
$$

Here $\boldsymbol{\Sigma}$ denotes the overall (symmetric) stress tensor, $\lambda(t)$ a time-varying, positive or negative scalar, and $\boldsymbol{\Sigma}_{0}$ a fixed, axisymmetric, symmetric tensor. The overall triaxiality $T$ is defined by

$$
T \equiv \frac{\Sigma_{m}}{\Sigma_{e q}}
$$

where $\Sigma_{m} \equiv \frac{1}{3} \operatorname{tr} \Sigma$ and $\Sigma_{e q} \equiv\left(\frac{3}{2} \Sigma^{\prime}: \Sigma^{\prime}\right)^{1 / 2}$ denote the overall mean and von Mises equivalent stresses $\left(\boldsymbol{\Sigma}^{\prime}\right.$ is the deviator of $\left.\boldsymbol{\Sigma}\right)$. Conditions (2) ensure constancy of this triaxiality in absolute value.

The porosity (void volume fraction) $f$ is defined by

$$
f \equiv \frac{\operatorname{vol}(\omega)}{\operatorname{vol}(\Omega)}=\frac{a^{3}}{b^{3}}
$$

where $\operatorname{vol}(\omega)$ denotes the volume of the void and $\operatorname{vol}(\Omega)$ that of the cell (including the void). It is studied as a function of the overall algebraic (signed) equivalent strain $\bar{E}_{e q}$. This quantity is defined by the formula

$$
\bar{E}_{e q} \equiv \int_{0}^{t} \operatorname{sgn}\left(\Sigma_{m}(\tau)\right) D_{e q}(\tau) d \tau
$$

where $\operatorname{sgn}(x)$ denotes the sign of $x$ and $D_{e q} \equiv\left(\frac{2}{3} \mathbf{D}^{\prime}: \mathbf{D}^{\prime}\right)^{1 / 2}$ the overall von Mises equivalent strain rate $\left(\mathbf{D}^{\prime}\right.$ is the deviator of $\left.\mathbf{D}\right)$. Note that $\bar{E}_{e q}$ increases in tension but decreases back in compression, unlike the usual equivalent strain rate. Each cycle is composed of two semi-cycles. The first is tensile $\left(\Sigma_{m}>0, T>0\right)$ and involves an increase of $\bar{E}_{e q}$ from 0 to some prescribed maximum value $\bar{E}_{e q}^{\max }$. The second is compressive $\left(\Sigma_{m}<0, T<0\right)$ and involves a decrease of $\bar{E}_{e q}$ back from $\bar{E}_{e q}^{\max }$ to 0 .

The 2D, axisymmetric mesh used is represented in Figure 2 in its initial, undeformed configuration. The sole upper half of the structure is discretized, thanks to symmetry about the horizontal mid-plane. The mesh is made of 1640 bilinear selectively subintegrated quadrilaterals and 1681 nodes. The initial porosity is $f_{0}=10^{-3}$.

The elastic constants of the material considered are $E$ (Young's modulus) $=1,500 \mathrm{GPa}$ and $\nu$ (Poisson's ratio $)=0.3$. The value of $E$ is artificially enhanced so as to simulate a quasi-rigid material, following the remark made in the Introduction. Strain hardening is assumed to be purely isotropic. The stress-strain curve in uniaxial tension is that of the A508 Cl.3 steel used in nuclear components; it is represented in Figure 3. The yield stress is regarded as constant for equivalent strains larger than 0.85 (largest value considered in the figure).

At each step of the calculation, the volumes of the void and the cell are deduced from the positions of the nodes lying on the internal and external surfaces of the mesh, accounting 


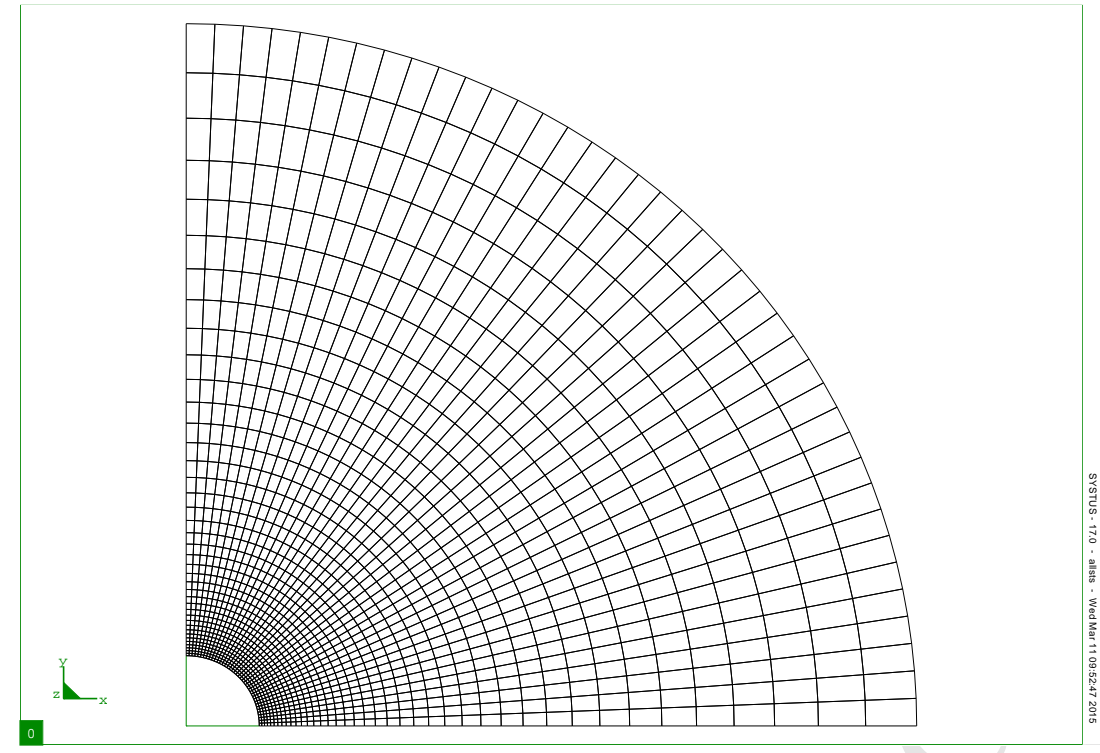

Fig. 2. Initial mesh of the hollow sphere $-f_{0}=10^{-3}$

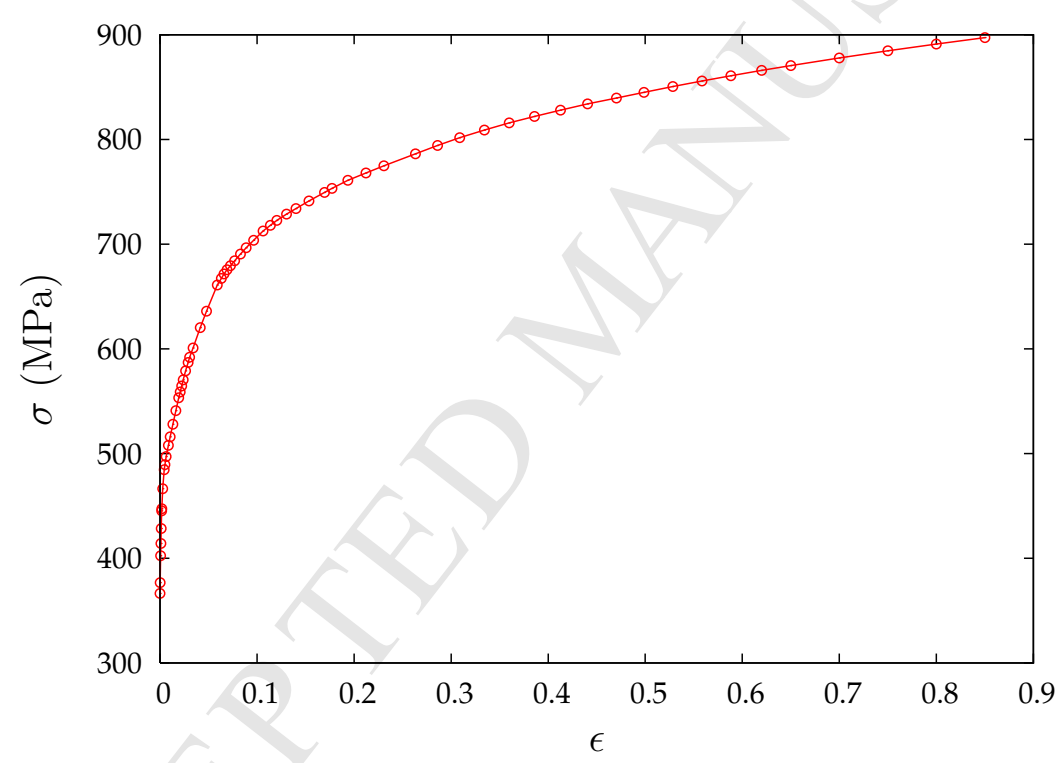

Fig. 3. Stress-strain curve of the A508 Cl.3 steel in uniaxial tension

for axisymmetry; the value of the porosity follows. The nonzero components of the overall stress tensor are obtained from the formula

$$
\Sigma_{i j}=\frac{1}{\operatorname{vol}(\Omega)} \int_{\Omega-\omega} \sigma_{i j}(\mathbf{x}) d \Omega
$$

where the $\sigma_{i j}$ denote the components of the local stress tensor. A dichotomy is used to adjust the value of the ratio $D_{x x} / D_{y y}$ so as to respect the prescribed value of the ratio $\Sigma_{x x} / \Sigma_{y y}$ within an accuracy of $10^{-3}$. This method is rustic but robust. In practice the prescribed value of $\Sigma_{x x} / \Sigma_{y y}$ is positive and smaller than unity, so that $\Sigma_{y y}>\Sigma_{x x}>0$ in tension and $\Sigma_{y y}<\Sigma_{x x}<0$ in compression: the largest overall principal stress in absolute value is the axial one, $\Sigma_{y y}$. Three values of the absolute value of the triaxiality are considered, $|T|=1,2$ and 3 . In view of the very different corresponding porosity rates, different maximum values of the overall algebraic equivalent strain $\bar{E}_{e q}^{\max }$ are considered 
for the various values of $|T|: \bar{E}_{e q}^{\max }=0.4,0.2$ and 0.08 for $|T|=1,2$ and 3 respectively. Four cycles are simulated in each case.

All computations use the Large Strain Plasticity option of the SYSTUS ${ }^{\circledR}$ finite element programme developed by ESI-Group.

\subsection{Results}

Figure 4 is included to facilitate understanding of the next, more essential ones. It considers the typical case $|T|=3$. First, Figure 4(a) displays the calculated evolution of the overall algebraic equivalent strain $\bar{E}_{e q}$ in time. During each cycle, this strain increases from 0 to some maximum value, then decreases back to 0. Second, Figure 4(b) displays the evolution of the normalized porosity $f / f_{0}$ in time. Again, during each cycle, this porosity increases from some minimum value to some maximum one, then decreases back. However the maximum reached increases with the number of cycles.

Figures 5, 6 and 7 combine such figures by eliminating time and now displaying the evolution of the normalized porosity $f / f_{0}$ directly as a function of the overall algebraic equivalent strain $\bar{E}_{e q}$. These figures are for the values $|T|=1,2,3$ respectively. A specific colour is used for each cycle for ease of identification. The ratcheting of the porosity is conspicuous in all cases. It is however much more important for the higher values of $|T|$, obviously because of the larger values of the porosity rate. In fact, the minimum value of $f$ is almost invariable from one cycle to the next one, but its maximum value gradually increases. This increase is an important feature since what governs the possible onset of coalescence of voids (leading to formation of a macroscopic crack) during one specific cycle is precisely the maximum porosity reached during this cycle.

A complementary simulation of the first two cycles has also been performed in the absence of strain hardening (ideal-plastic material), for the value $|T|=3$. Figure 8 compares the results of this simulation to those obtained when including it. There is almost no ratcheting of the porosity for the ideal-plastic material. This provides a double check:

- On the values of the elastic constants used. Indeed it has been noted in Section 2.1 that elasticity may generate a ratcheting of the porosity even in the absence of strain hardening. Absence of this ratcheting is therefore a confirmation that the value of Young's modulus used is high enough for the material to be safely considered as elastically quasi-rigid, as desired.

- On the control of the triaxiality. Indeed spurious variations of $|T|$ between the tensile and compressive parts of a cycle may generate some asymmetry between them. Therefore absence of ratcheting of the porosity, that is symmetry between the tensile and compressive parts of a cycle, implies that the accuracy of $10^{-3}$ used in the control of the ratio $\Sigma_{x x} / \Sigma_{y y}$ is small enough to warrant absence of such variations. 


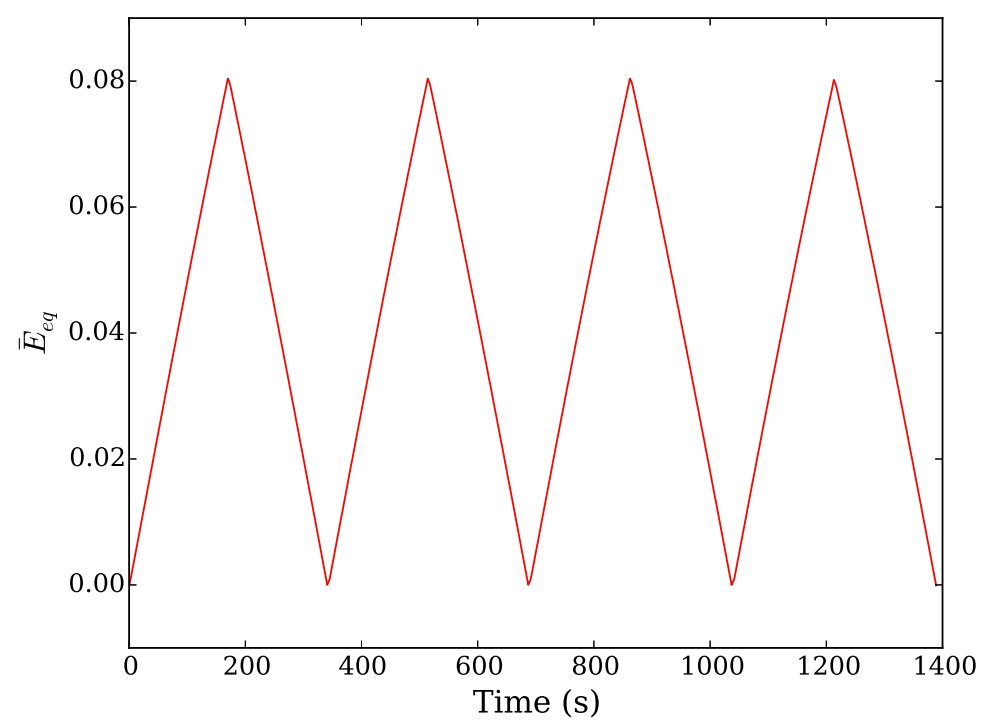

(a) Algebraic equivalent strain

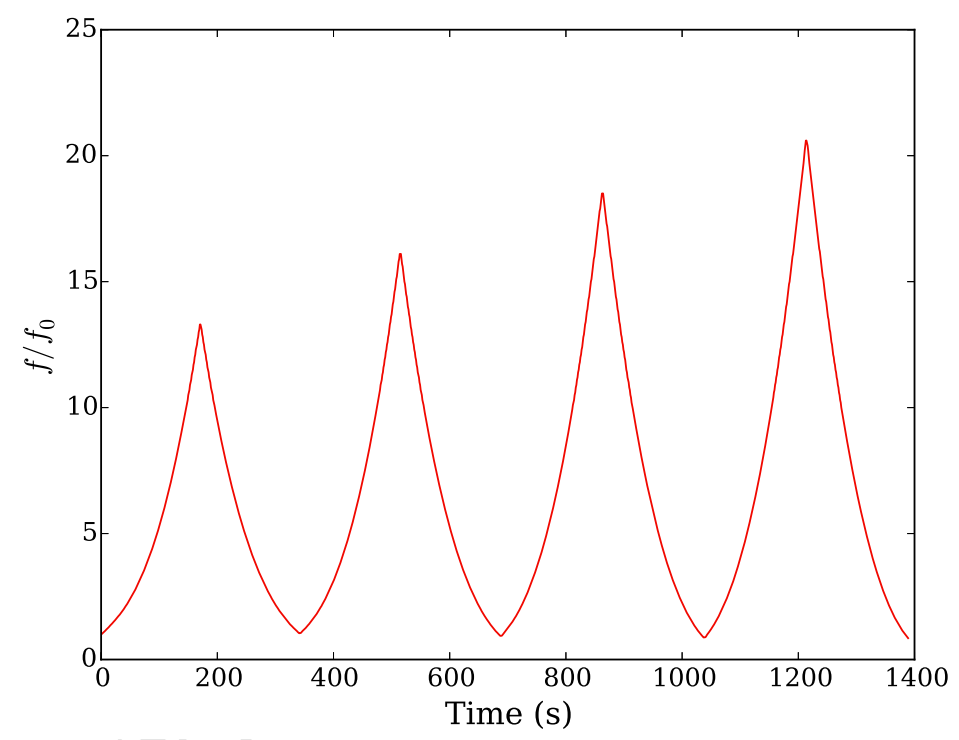

(b) Normalized porosity

Fig. 4. Evolutions of the algebraic equivalent strain and the normalized porosity as functions of time - Numerical simulation, $|T|=3$

\section{Absence of ratcheting of the porosity in Gurson's original model}

The first step in the theoretical analysis of the ratcheting of the porosity is to note that Gurson (1977)'s homogenized model, in its original form, fails to predict such an effect. This stems from the following property (Devaux et al., 1997):

Consider an elementary porous cell made of some rigid-hardenable material and subjected to some cyclically, proportionally varying overall stress tensor $\boldsymbol{\Sigma}(t)$. Let $f_{0}$ denote the initial porosity and $|T|$ the prescribed, constant absolute value of the triaxiality. Gurson 


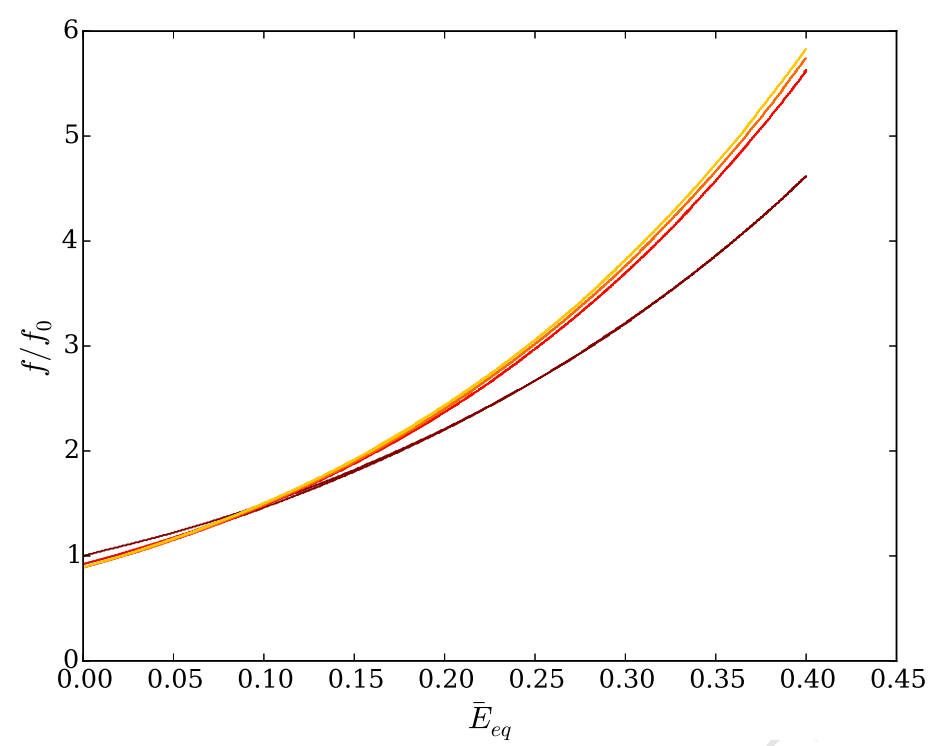

Fig. 5. Evolution of the normalized porosity as a function of the overall algebraic equivalent strain - Numerical simulation, $|T|=1$ - Cycle 1: violet; 2: red; 3: orange; 4: yellow

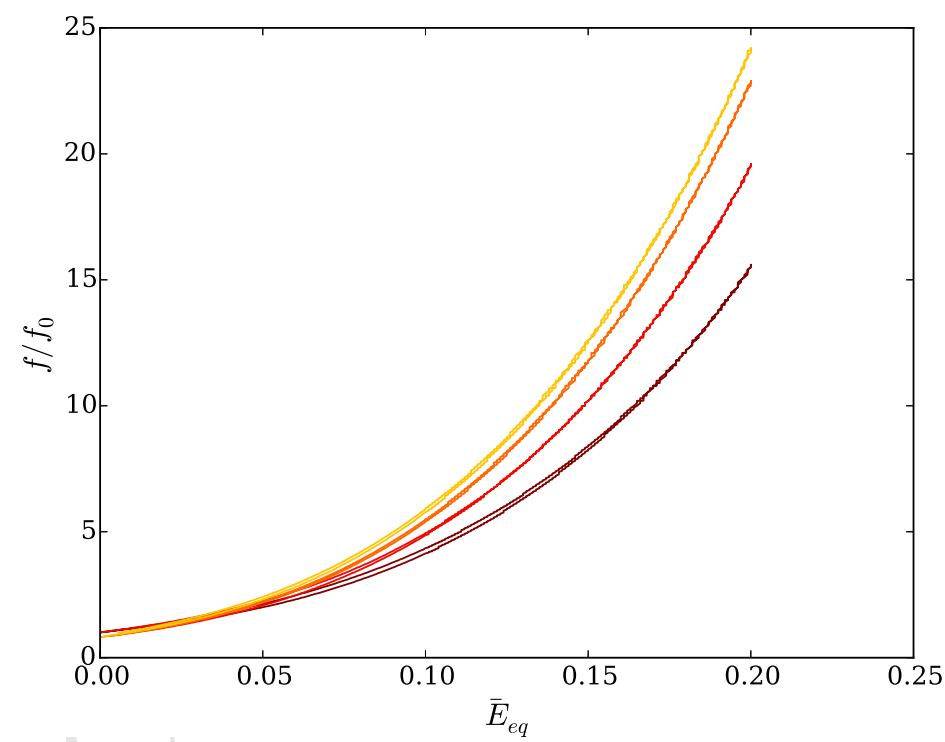

Fig. 6. Evolution of the normalized porosity as a function of the overall algebraic equivalent strain - Numerical simulation, $|T|=2$ - Cycle 1: violet; 2 : red; 3: orange; 4: yellow

(1977)'s model, when applied to this cell considered as a homogeneous volume element, asserts that its present porosity $f$ is a function of the sole parameters $f_{0}$ and $|T|$ plus the algebraic equivalent strain $\bar{E}_{\text {eq }}$ defined by equation (5).

A consequence of this property is that the curve composed of the successive points $\left(\bar{E}_{\text {eq }}, f\right)$ must be identical for all successive semi-cycles, since these semi-cycles all have the same values of $f_{0}$ and $|T|$. This means that the evolution of the porosity must be stabilized right from the first semi-cycle, which (erroneously) rules out ratcheting.

The proof of the property announced consists of three steps. In a first step, start from the 


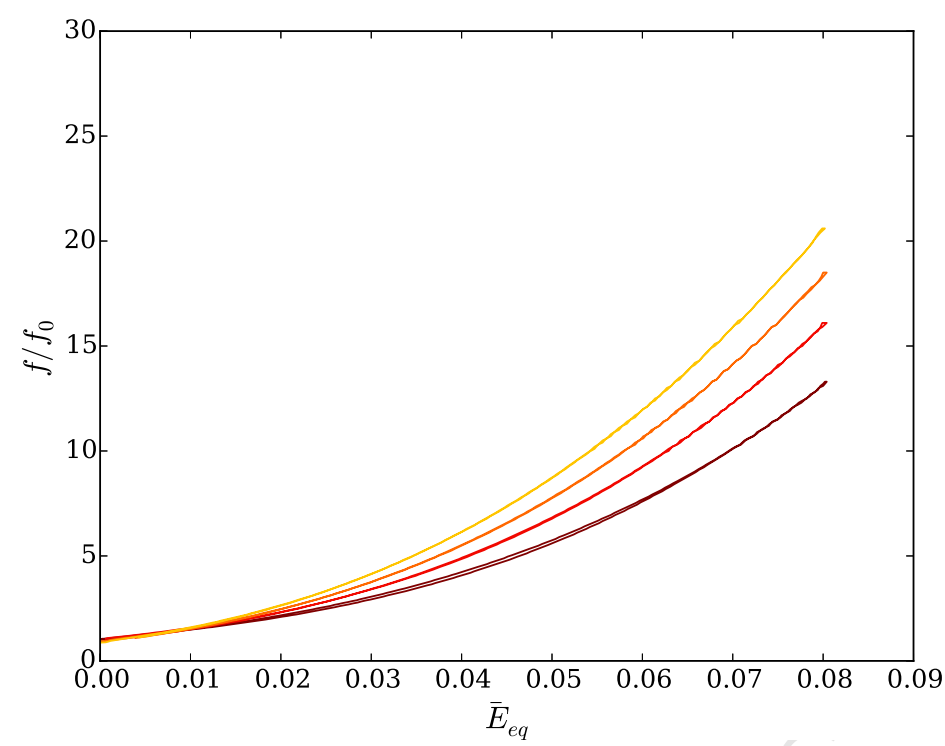

Fig. 7. Evolution of the normalized porosity as a function of the overall algebraic equivalent strain - Numerical simulation, $|T|=3$ - Cycle 1: violet; 2 : red; 3: orange; 4: yellow

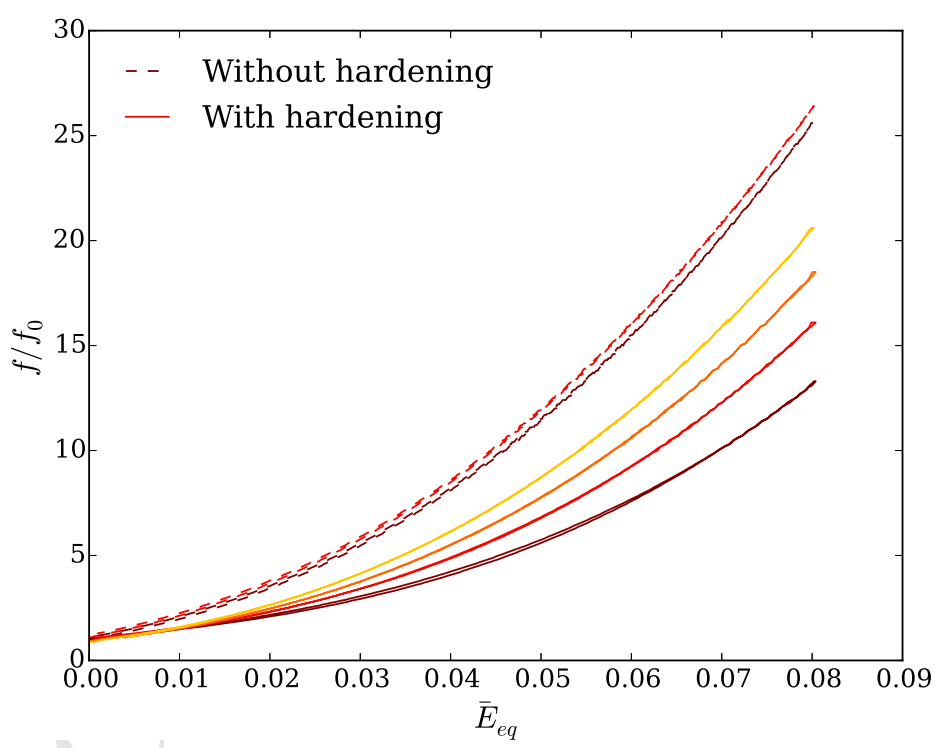

Fig. 8. Comparison of the evolutions of the normalized porosity with and without strain hardening - Numerical simulation, $|T|=3$ - Cycle 1: violet; 2: red; 3: orange; 4: yellow

expression of Gurson (1977)'s criterion,

$$
\frac{\Sigma_{e q}^{2}}{\bar{\sigma}^{2}}+2 q f \cosh \left(\frac{3}{2} \frac{\Sigma_{m}}{\bar{\sigma}}\right)-1-q^{2} f^{2}=0,
$$

where $\bar{\sigma}$ denotes some "average yield stress" of the matrix and $q$ Tvergaard (1981)'s famous parameter. Rewrite this expression in the form

$$
X^{2}+2 q f \cosh \left(\frac{3}{2}|T| X\right)-1-q^{2} f^{2}=0 \quad, \quad X \equiv \frac{\Sigma_{e q}}{\bar{\sigma}}
$$


( $X$ is a dimensionless "reduced equivalent stress"). Equation (7) implicitly defines $X$ as a function of the sole variables $|T|$ and $f$ :

$$
X \equiv \Phi(|T|, f)
$$

In a second step, combine the evolution law of the porosity and the (normal) flow rule to get

$$
\begin{aligned}
\dot{f}=(1-f) \operatorname{tr} \mathbf{D} & =\frac{3 q f(1-f)}{2} \frac{\bar{\sigma}}{\Sigma_{e q}} \sinh \left(\frac{3}{2} \frac{\Sigma_{m}}{\bar{\sigma}}\right) D_{e q} \\
& =\frac{3 q f(1-f)}{2 X} \sinh \left(\frac{3}{2}|T| X\right) \operatorname{sgn}\left(\Sigma_{m}\right) D_{e q}
\end{aligned}
$$

This implies that

$$
\frac{\dot{f}}{\operatorname{sgn}\left(\Sigma_{m}\right) D_{e q}}=\frac{d f}{d \bar{E}_{e q}}=\frac{3 q f(1-f)}{2 X} \sinh \left(\frac{3}{2}|T| X\right)
$$

so that the derivative $d f / d \bar{E}_{e q}$ depends only on $|T|, f$ and $X$. But $X$ depends itself only on $|T|$ and $f$ by equation (8). It follows that $d f / d \bar{E}_{e q}$ depends only on $|T|$ and $f$ :

$$
\frac{d f}{d \bar{E}_{e q}} \equiv \Psi(|T|, f)
$$

The third, final step consists of integrating the differential equation (9) with respect to $\bar{E}_{e q}$. Separation of variables yields

$$
\frac{d f}{\Psi(|T|, f)}=d \bar{E}_{e q} \Rightarrow \int_{f_{0}}^{f} \frac{d f^{\prime}}{\Psi\left(|T|, f^{\prime}\right)}=\bar{E}_{e q} .
$$

Equation $(10)_{2}$ shows that $f$ depends only on $f_{0},|T|$ and $\bar{E}_{e q}$, as announced.

Detailed inspection of this proof reveals that it does not depend on the details of Gurson (1977)'s expression of the yield function; nor does it depend on the evolution law of the average yield stress $\bar{\sigma} .{ }^{3}$ In fact the only thing that really matters is that the effect of strain hardening is summarized within a single internal variable $\bar{\sigma}$, which permits to express the yield function in terms of some "reduced stress tensor" $\boldsymbol{\Sigma} / \bar{\sigma}$. This means that any yield criterion possessing the same feature of "reduction" of strain hardening effects to a single internal variable, will lead to the same (erroneous) conclusion of absence of ratcheting of the porosity under cyclic loadings.

\section{The original LPD model}

We now look for some homogenized description of porous ductile materials accounting for the ratcheting of the porosity. In this search the remark just made leaves no choice but to

$\overline{3}$ This is apparent in the fact that the precise form of the function $\Psi$ is immaterial. 
consider a modelling of strain hardening effects involving at least two internal variables. The LPD model proposed by Leblond et al. (1995) is of this type and is briefly reviewed here.

Leblond et al. (1995)'s proposed overall yield function is a straightforward extension of that of Gurson (1977):

$$
\frac{\Sigma_{e q}^{2}}{\Sigma_{1}^{2}}+2 q f \cosh \left(\frac{3}{2} \frac{\Sigma_{m}}{\Sigma_{2}}\right)-1-q^{2} f^{2}=0
$$

In this expression $\Sigma_{1}$ and $\Sigma_{2}$ are macroscopic internal variables connected to the distribution of the local strain hardening within the heterogeneously deformed matrix. To determine these internal variables, Leblond et al. (1995) used an approximate analytical solution to some typical problem: namely, a hollow sphere made of some rigid-hardenable material and subjected to some arbitrary loading through conditions of homogeneous boundary strain rate. ${ }^{4}$

Leblond et al. (1995)'s approximate solution accounted for the geometry changes due to the sole hydrostatic part of the loading. Thus the cell was considered to approximately remain spherical. The present inner radius $a$ and outer radius $b$ of this sphere, and the radial position $r$ of the current point, were connected to the corresponding initial quantities $A, B$ and $R$ through the following relations resulting from incompressibility:

$$
a^{3}-A^{3}=b^{3}-B^{3}=r^{3}-R^{3} \equiv \Delta \omega .
$$

In this equation the quantity $\Delta \omega$ represents, up to a factor of $4 \pi / 3$, the additional void volume and is given by

$$
\Delta \omega \equiv \frac{f-f_{0}}{1-f} B^{3}
$$

The derivation of the expressions of the internal variables $\Sigma_{1}$ and $\Sigma_{2}$ then included two steps, each of which involved extra approximations:

(1) The determination of $\Sigma_{1}$ and $\Sigma_{2}$ as functions of the distribution of the local yield stress $\sigma\left(\epsilon_{e q}\right)$ within the hollow sphere. The quantity $\epsilon_{e q} \equiv \int_{0}^{t} d_{e q}(\tau) d \tau$ here denotes the local cumulated equivalent strain $\left(d_{e q} \equiv\left(\frac{2}{3} \mathbf{d}: \mathbf{d}\right)^{1 / 2}\right.$ is the local von Mises equivalent strain rate).

(2) The determination of the distributions of $\epsilon_{e q}$ and $\sigma\left(\epsilon_{e q}\right)$ within the hollow sphere resulting from the previous mechanical history.

In Step (1), expressions of $\Sigma_{1}$ and $\Sigma_{2}$ were deduced from estimations of the overall yield stresses of the hollow sphere under purely deviatoric and purely hydrostatic loadings,

$\overline{4}$ It is worth noting that Gurson (1977) developed his homogenization procedure for ideal-plastic materials only; the approach he subsequently adopted to introduce strain hardening was purely heuristic. In contrast, Leblond et al. (1995) sticked to micromechanics by extending Gurson (1977)'s homogenization procedure to the hardenable case. 
respectively. These expressions were as follows:

$$
\Sigma_{1}=\frac{1}{b^{3}-a^{3}} \int_{a^{3}}^{b^{3}} \sigma\left(\left\langle\epsilon_{e q}\right\rangle_{\mathcal{S}(r)}\right) d\left(r^{3}\right) \quad ; \quad \Sigma_{2}=\frac{1}{\ln \left(b^{3} / a^{3}\right)} \int_{a^{3}}^{b^{3}} \sigma\left(\left\langle\epsilon_{e q}\right\rangle_{\mathcal{S}(r)}\right) \frac{d\left(r^{3}\right)}{r^{3}} .
$$

In this equation the symbol $\langle\cdot\rangle_{\mathcal{S}(r)}$ represents an average value over the spherical surface $\mathcal{S}(r)$ of radius $r$, and $\left\langle\sigma\left(\epsilon_{e q}\right)\right\rangle_{\mathcal{S}(r)}$ has approximately been replaced with $\sigma\left(\left\langle\epsilon_{e q}\right\rangle_{\mathcal{S}(r)}\right)$.

Clearly, as soon as the function $\sigma\left(\epsilon_{e q}\right)$ is not a constant, the values of $\Sigma_{1}$ and $\Sigma_{2}$ are distinct. More precisely $\Sigma_{2}$ is larger than $\Sigma_{1}$; indeed the logarithmic measure $d\left(r^{3}\right) / r^{3}$ puts more "weight" on the vicinity of the void's boundary, where strain hardening is maximum, than the standard measure $d\left(r^{3}\right)$.

The basis of Step (2) was the following expression of the time-derivative of $\left\langle\epsilon_{e q}\right\rangle_{\mathcal{S}(r)}$ :

$$
\frac{d}{d t}\left[\left\langle\epsilon_{e q}\right\rangle_{\mathcal{S}(r)}\right]=\left\langle d_{e q}\right\rangle_{\mathcal{S}(r)} \simeq \sqrt{\left\langle d_{e q}^{2}\right\rangle_{\mathcal{S}(r)}} \simeq \sqrt{4 \frac{b^{6}}{r^{6}} D_{m}^{2}+D_{e q}^{2}}
$$

where $D_{m} \equiv \frac{1}{3} \operatorname{tr} \mathbf{D}$ represents the overall mean strain rate. The final approximation here results from use of Gurson (1977)'s trial velocity fields.

To integrate this expression analytically in time, it is necessary to introduce some hypothesis on the evolution of the ratio $D_{m} / D_{e q}$. Leblond et al. (1995) made the assumption of positively proportional straining: $\mathbf{D}(t)=\mu(t) \mathbf{D}_{0}$ where $\mu(t)$ is a positive time-varying scalar and $\mathbf{D}_{0}$ a fixed symmetric tensor. This hypothesis implied constancy of the ratio $D_{m} / D_{e q}$ and led to a somewhat complex expression of $\left\langle\epsilon_{e q}\right\rangle_{\mathcal{S}(r)}$, which need not be recalled here.

As mentioned in the Introduction, Devaux et al. (1997) compared the results of their micromechanical simulations of the ratcheting of the porosity to the predictions of the LPD model, in the form just expounded. The results were mitigated. This may be due to several factors but one explanation certainly lies in the hypothesis of positively proportional straining made in the model. Such a hypothesis does not apply to cyclic loadings since each component of the overall strain rate changes sign from one semi-cycle to the next one. ${ }^{5}$

Some comments pertaining to the work of Gao et al. (1998) are finally in order here. In this work the authors showed that the void growth rate depends on the hardening law of the material. More specifically, adopting Gurson (1977)'s model with Tvergaard (1981)'s $q$-parameter, they determined the "optimal" value of this parameter and concluded that this optimal value depends on the hardening exponent. This conclusion bears several connections with the problem investigated here:

- There is a first connection with the earlier work of Leblond et al. (1995) where the LPD model was proposed. Indeed Leblond et al. (1995) reviewed Koplik and Needleman (1988)'s numerical simulations of elementary porous cells loaded under conditions

5 Admittedly Leblond et al. (1995) did not have such loadings in mind when developing their model. 
of positive proportional stressing. They noted that the curve composed of the points $\left(E_{e q}, f\right)$ does not only depend upon the initial porosity and the fixed triaxiality, but also on the hardening curve of the material. Gao et al. (1998)'s conclusion that void growth is sensitive to strain hardening was just the same statement expressed in different words.

- There is a second, more subtle connection with the phenomenon of ratcheting of the porosity under cyclic loadings. Indeed during such loadings strain hardening varies, and even saturates after some degree of deformation. Combining this observation with that of Gao et al. (1998) that the porosity rate depends on strain hardening, one concludes that this rate must be different during the first cycles and the last ones. In other words, Gao et al. (1998)'s observation implicitly entails the impossibility of stabilization of the porosity evolution during cyclic loadings.

- Finally, although Gao et al. (1998)'s suggested modification of Gurson (1977)'s model - use a $q$-parameter depending on the hardening law - differs from that proposed by Leblond et al. (1995) and adopted here - introduce different internal variables $\Sigma_{1}$ and $\Sigma_{2}$ in the square and the hyperbolic cosine -, the two are related. Indeed in both cases the effect of strain hardening appears in two parameters ( $\bar{\sigma}$ and $q$, or $\Sigma_{1}$ and $\left.\Sigma_{2}\right)$ instead of being concentrated in a single one. Gao et al. (1998)'s suggested model is probably a viable alternative to the LPD model presented above and improved below. But it is also probably more cumbersome to use. Indeed in Gao et al. (1998)'s model the q-parameter must be calibrated for each hardening law, perhaps even as a function of the cumulated equivalent strain, whereas it is a mere easy-to-calibrate constant in the LPD model.

\section{$5 \quad$ An improved LPD model for cyclic loadings}

\subsection{Presentation of the model}

Following the remark just made, we propose to retain all elements of the LPD model except for the hypothesis of positively proportional straining. In fact, no hypothesis is made on the evolution of the ratio $D_{m} / D_{e q}$ in time. Analytical integration of equation (15) then becomes impossible, and numerical integration becomes necessary. One drawback is the necessity of considering, at each "macroscopic material point", an underlying spherical "microcell" discretized radially, and storing the values of $\left\langle\epsilon_{e q}\right\rangle_{\mathcal{S}(r)}$ as internal variables at the discretization points. But the development of computer storage capabilities makes such a procedure quite feasible nowadays, provided that the microcell is discretized radially with a reasonable number (say $\lesssim 100$ ) of points.

Thus, at each material point, the initial spherical microcell is discretized using $N+1$ points:

$$
A=R_{0}<R_{1}<\ldots<R_{N-1}<R_{N}=B .
$$

One also considers the midpoints of the segments $\left[R_{i-1}, R_{i}\right]$ :

$$
R_{i}^{\prime} \equiv \frac{1}{2}\left(R_{i-1}+R_{i}\right) \quad(i=1, \ldots, N)
$$


At an arbitrary instant, the current positions $r_{i}, r_{i}^{\prime}$ of the points $R_{i}$ and $R_{i}^{\prime}$ are

$$
r_{i} \equiv\left(R_{i}^{3}+\Delta \omega\right)^{1 / 3}(i=0,1, \ldots, N) \quad ; \quad r_{i}^{\prime} \equiv\left({R_{i}^{\prime}}^{3}+\Delta \omega\right)^{1 / 3}(i=1, \ldots, N) .
$$

The additional void volume $\Delta \omega$ here is given by equation (13). The internal variables to be stored are the average values $\left\langle\epsilon_{e q}\right\rangle_{\mathcal{S}\left(r_{i}^{\prime}\right)}$ of the cumulated equivalent strain $\epsilon_{e q}$ over the various spherical surfaces of radius $r_{i}^{\prime}$, hereafter denoted $\left\langle\epsilon_{e q}\right\rangle_{i}$ for shortness.

The quantities $\Sigma_{1}$ and $\Sigma_{2}$ are then given in terms of the internal variables $\left\langle\epsilon_{e q}\right\rangle_{i}$ by the following discretized equivalents of expressions (14):

$$
\Sigma_{1}=\frac{1}{b^{3}-a^{3}} \sum_{i=1}^{N} \sigma\left(\left\langle\epsilon_{e q}\right\rangle_{i}\right)\left(r_{i}^{3}-r_{i-1}^{3}\right) ; \Sigma_{2}=\frac{1}{\ln \left(b^{3} / a^{3}\right)} \sum_{i=1}^{N} \sigma\left(\left\langle\epsilon_{e q}\right\rangle_{i}\right)\left[\ln \left(r_{i}^{3}\right)-\ln \left(r_{i-1}^{3}\right)\right] .
$$

Also, the evolution in time of the internal variables $\left\langle\epsilon_{e q}\right\rangle_{i}$ is governed by equation (15) which takes the form

$$
\frac{d}{d t}\left\langle\epsilon_{e q}\right\rangle_{i}=\sqrt{4 \frac{b^{6}}{r_{i}^{\prime 6}} D_{m}^{2}+D_{e q}^{2}}
$$

Equations $(19,20)$ permit to calculate the evolution of the internal variables $\left\langle\epsilon_{e q}\right\rangle_{i}, \Sigma_{1}$ and $\Sigma_{2}$ during the whole mechanical history.

\subsection{Comparison with the results of the micromechanical simulations}

The comparison of numerical and theoretical results for the ratcheting of the porosity requires to make a choice for Tvergaard (1981)'s q-parameter to be used in the improved LPD model.

Tvergaard (1981) introduced this parameter to bring the predictions of the original Gurson model to closer agreement with the results of various micromechanical simulations of plastic porous media. He interpreted the necessity of this adjustment as a result of the difference between the spherical cell geometry considered by Gurson (1977) - for which $q$ was unity - and the more realistic geometries he himself considered. The choice $q=1$ therefore seems appropriate in the present context; indeed the micromechanical simulations of Section 2 were precisely performed for a spherical cell like in Gurson (1977)'s work.

However, Gurson (1977)'s approximate homogenization procedure was very recently reconsidered by Leblond and Morin (2014). Upon refinement of an approximation made by Gurson, these authors concluded that even for a spherical cell, his model requires introduction of a slightly non-unity value of $q$ to accurately predict the porosity rate. The optimum value depends weakly upon the triaxiality. It may be estimated from Leblond and Morin (2014)'s Figure 3 to be of the order of 1.13 for $T=1,1.08$ for $T=2$ and 1 for $T=3$. These values are therefore adopted in the comparisons to follow.

The calculations based on the homogenized model are for a homogeneous volume element, so that elementary "0D" simulations would be sufficient. However future applications 
of the LPD model, in its extended form, to cyclic loading of complex components are envisaged. With these in mind, the model has been implemented into the SYSTUS ${ }^{\circledR}$ general purpose finite element programme developed by ESI-Group. The algorithm for the plastic correction of the elastic stress predictor ("projection" of this predictor onto the LPD yield locus) is very similar to that proposed by Enakoutsa et al. (2007) for Gurson (1977)'s model. It will therefore not be detailed here. The results given below have been obtained by radially discretizing the spherical microcells with $N+1=41$ points defining 40 thin spherical crowns. This exactly corresponds to the number of elements in the radial direction in the micromechanical simulations. This discretization has been checked to be fine enough to warrant highly accurate evaluations of $\Sigma_{1}$ and $\Sigma_{2}$ through numerical integration.

Figures 9, 10 and 11 compare the evolutions of $f$ versus $\bar{E}_{e q}$ obtained in the micromechanical simulations and predicted by the improved LPS model. The values of $|T|$ are 1,2 and 3 in these three figures respectively. Again, different colours are used for the various cycles. The agreement between the simulations and the model is acceptable for $|T|=1$, good for $|T|=2$ and excellent for $|T|=3$. The looser agreement observed for $|T|=1$ does not really matter since the increase of the porosity is relatively small in this case. The almost perfect agreement observed for $|T|=3$ is in fact not surprising. Indeed for such a high value of $|T|$ the loading does not differ much from a purely hydrostatic one, for which the LPD model in its improved form provides the exact solution.

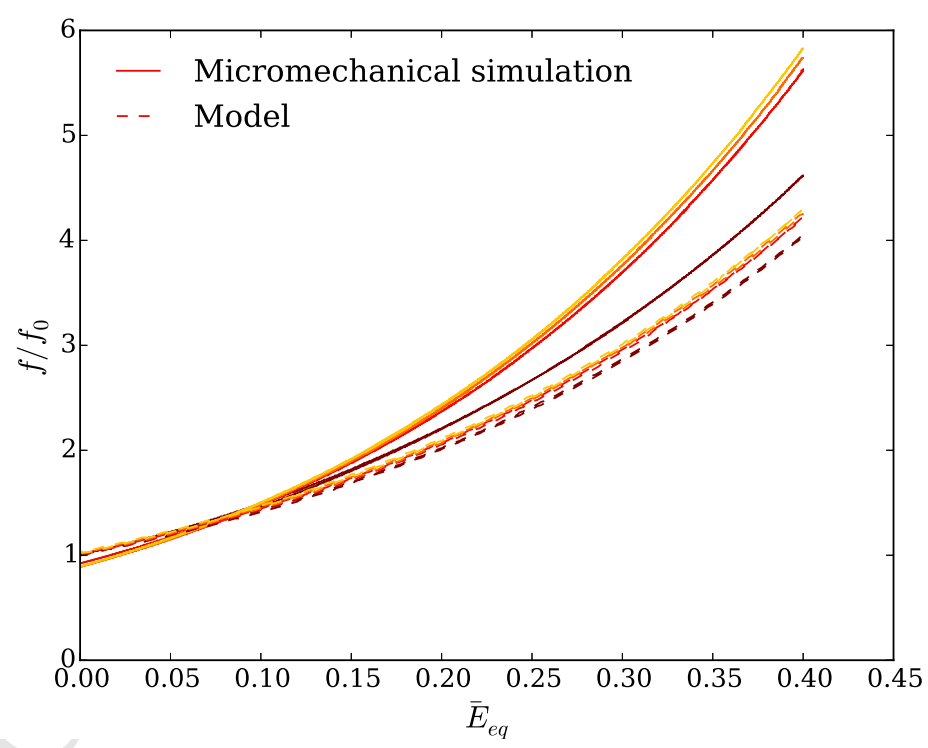

Fig. 9. Comparison of micromechanical simulations and model predictions for the evolution of the normalized porosity - $|T|=1, q=1.13$ - Cycle 1: violet; 2: red; 3: orange; 4: yellow

Figures 12 to 15 provide complementary checks on the validity of the model. They consider only the more interesting values 2 and 3 of $|T|$. First, figures 12 and 13 show the calculated distribution of the cumulated equivalent strain on the current, deformed configuration of the sphere. The instant selected corresponds to the end of the 7-th semi-cycle, when the porosity reaches its absolute maximum. Note the very high levels of straining reached, due to the strain concentration around the void. For $|T|=2$, the hypothesis of persistent sphericity made by the model is reasonably well satisfied. Also, the cumulated equivalent 


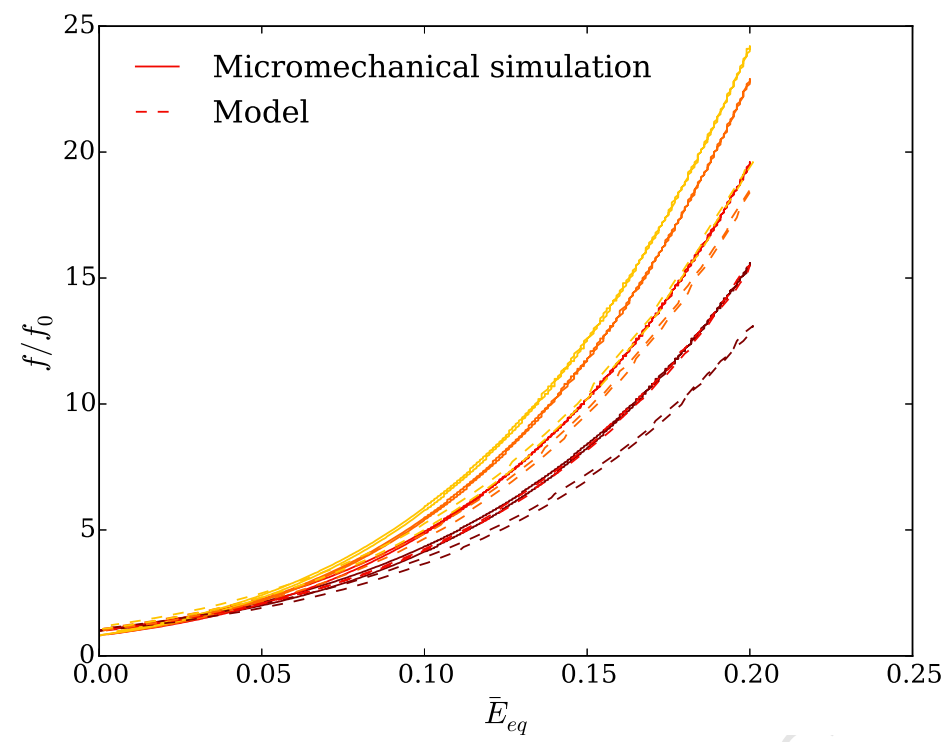

Fig. 10. Comparison of micromechanical simulations and model predictions for the evolution of the normalized porosity - $|T|=2, q=1.08$ - Cycle 1: violet; 2 : red; 3: orange; 4: yellow

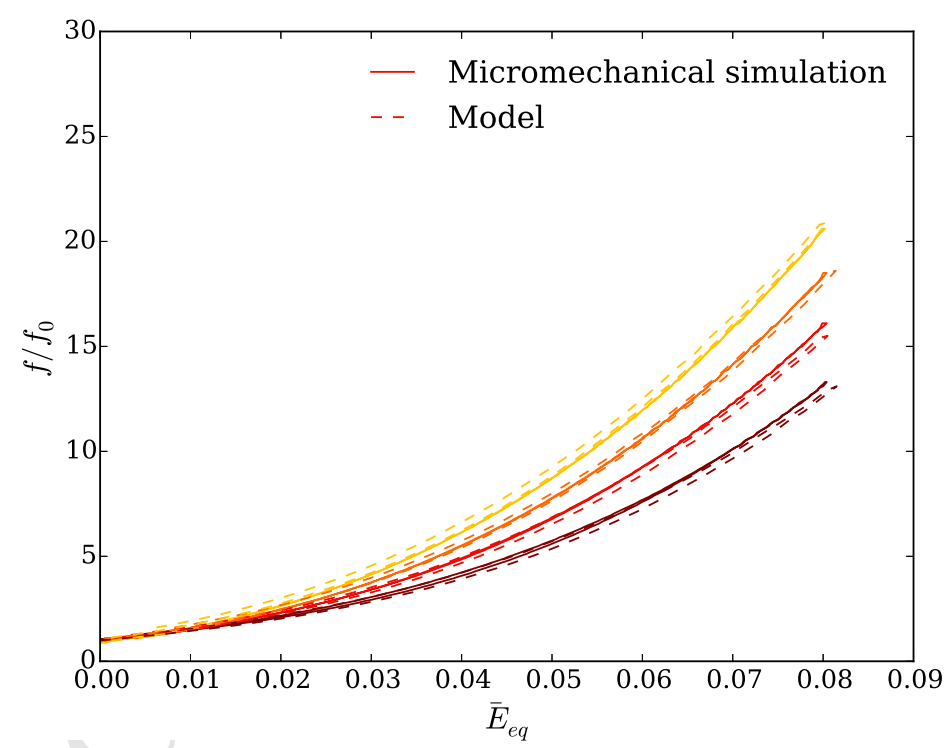

Fig. 11. Comparison of micromechanical simulations and model predictions for the evolution of the normalized porosity - $|T|=3, q=1$ - Cycle 1: violet; 2: red; 3: orange; 4: yellow

strain varies little in the orthoradial direction. This justifies the replacement of $\left\langle\sigma\left(\epsilon_{e q}\right)\right\rangle_{\mathcal{S}(r)}$ with $\sigma\left(\left\langle\epsilon_{e q}\right\rangle_{\mathcal{S}(r)}\right)$ in the expressions (14) of $\Sigma_{1}$ and $\Sigma_{2}$. For $|T|=3$ the two properties are also satisfied, with a much better degree of accuracy.

Figures 14 and 15 show the radial distribution of the average value of the von Mises equivalent strain rate over the spherical surface of radius $r$. Again, the instant selected corresponds to the end of the 7 -th semi-cycle. The values obtained in the micromechanical simulations are compared to those predicted by the theoretical formula (15). This formula provides an acceptable approximation for $|T|=2$, and an excellent one for $|T|=3$. 


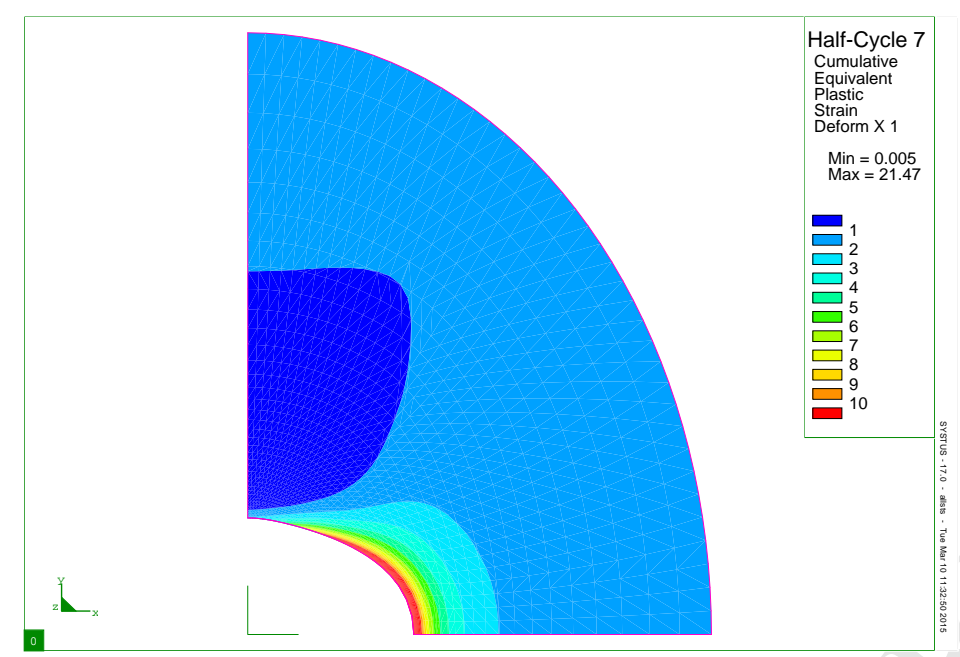

Fig. 12. Distribution of the equivalent cumulated strain on the deformed mesh at the end of the 7-th semi-cycle - $|T|=2$

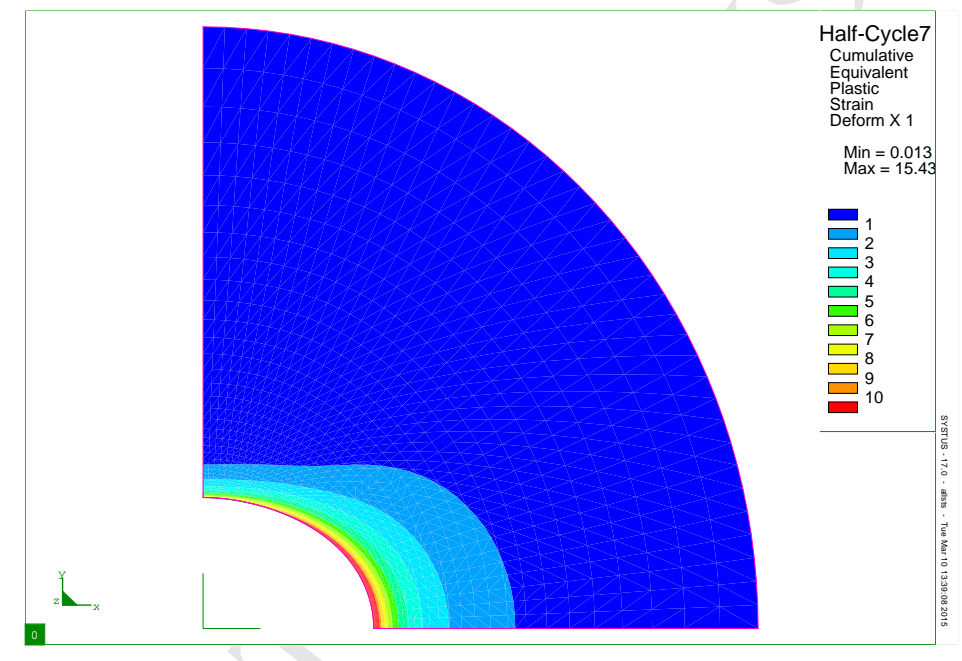

Fig. 13. Distribution of the equivalent cumulated strain on the deformed mesh at the end of the 7-th semi-cycle - $|T|=3$

\section{Conclusion}

The aim of this work was twofold. The first goal was to perform micromechanical simulations of the ratcheting of the porosity in elementary porous cells subjected to cyclic loadings. The second one was to propose a homogenized, Gurson-type model incorporating this effect; such a model should account for the currently observed reduction of ductility under cyclic loadings.

The numerical simulations considered an initially spherical cell containing an initially spherical void. This cell was loaded cyclically through conditions of homogeneous boundary strain rate. A constant absolute value of the triaxiality was imposed throughout. These simulations differed from previous ones essentially through the spherical shape of the cell and consideration of a very high Young's modulus. These differences were justified 


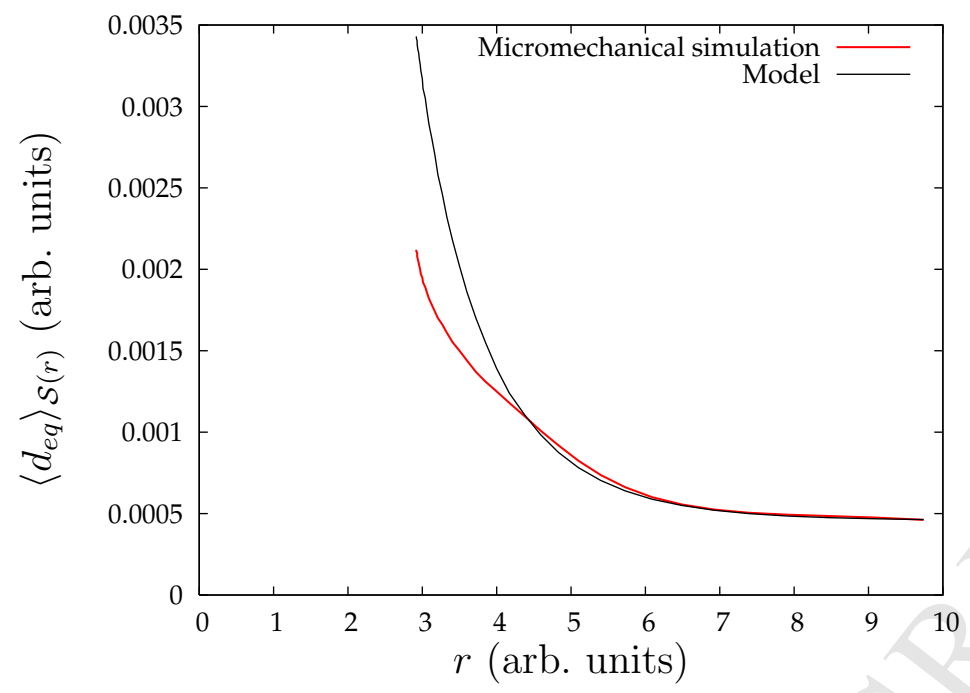

Fig. 14. Radial variation of the average value $\left\langle d_{e q}\right\rangle_{\mathcal{S}(r)}$ at the end of the 7 -th semi-cycle $-|T|=2$

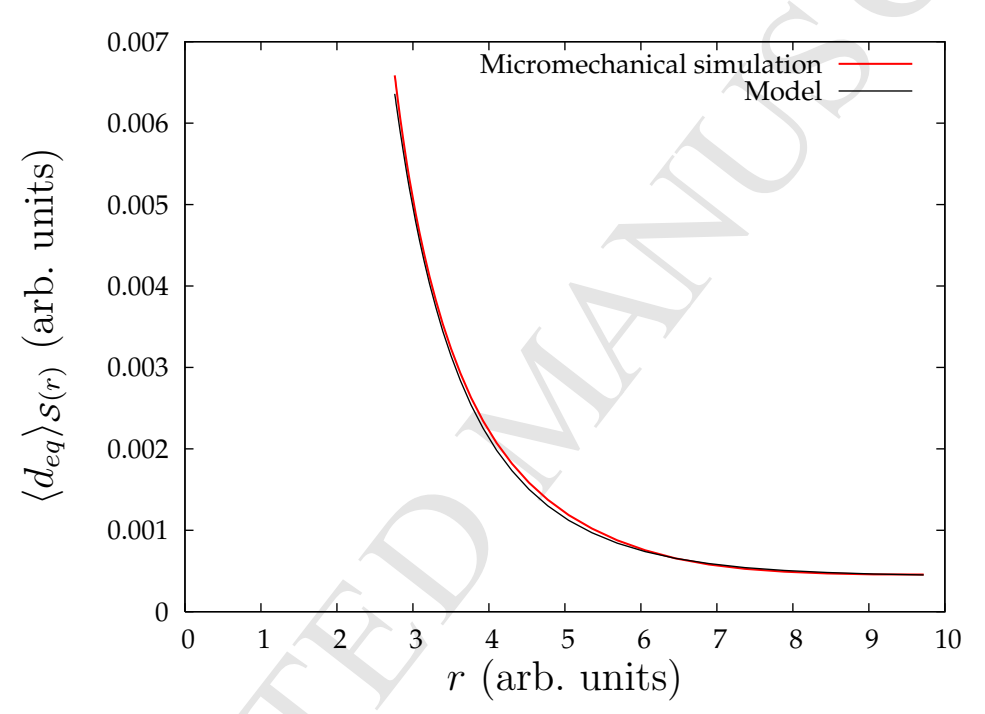

Fig. 15. Radial variation of the average value $\left\langle d_{e q}\right\rangle_{\mathcal{S}(r)}$ at the end of the 7 -th semi-cycle $-|T|=3$

by the wish to perform simulations complying better with the hypotheses made in the theoretical derivation of Gurson-type models. The results fully confirmed the existence of a ratcheting of the porosity under cyclic loadings. They also pointed out the decisive influence of strain hardening upon the phenomenon.

It was then shown, as a first step in the theoretical analysis of the ratcheting of the porosity, that Gurson (1977)'s model, in its original form, fails to reproduce such an effect. The key point in this failure is Gurson (1977)'s assumption that the effect of strain hardening may be summarized in a single macroscopic internal variable, representing some "average yield stress" of the heterogeneously deformed matrix.

The LPD model was then briefly described. This variant of Gurson (1977)'s model, due to Leblond et al. (1995), is an a priori good candidate for the correct theoretical description of the ratcheting of the porosity. Indeed it drops Gurson's introduction of a unique macroscopic internal variable to represent strain hardening effects, and introduces instead two distinct internal variables in the "square" and "cosh" terms of Gurson's yield function. 
When applied to elementary volumes subjected to cyclic loadings, the LPD model makes a better job than Gurson's model. However its predictions are still not quite satisfactory. One explanation of this mitigated success lies in the hypothesis of positively proportional straining made by the model. This hypothesis does not apply to cyclic loadings.

We finally depicted an improved version of the LPD model discarding this hypothesis. In this version it becomes impossible to analytically integrate, at the various points of the spherical "microcell" associated to each macroscopic material point, the evolution equation of the cumulated equivalent strain. Numerical integration requires radial discretization of the microcell, and storage of additional internal variables, namely the values of the local cumulated equivalent strain at the discretization points. The storage of a nottoo-large number of internal variables is quite feasible in view of present-day computer capabilities. A very good comparison was observed between the results of the micromechanical simulations and the predictions of the LPD model, in this improved version.

Future developments of the work will include:

- Further numerical micromechanical simulations for more hardenable, stainless steels. Such simulations are desirable since strain hardening is the major factor governing the ratcheting of the porosity under cyclic loadings.

- Applications of the LPD model, in its improved version, to finite element studies of ductile rupture of full-scale specimens or structures loaded cyclically.

- Extension of the work to matrices exhibiting kinematic or mixed isotropic/kinematic hardening.

\section{Acknowledgement}

The authors wish to express their warm thanks to the AREVA Company, and especially Drs. Stéphane Chapuliot and Philippe Gilles, for their continued moral and financial support of this work.

\section{References}

Besson J. and Guillemer-Neel C. (2003). An extension of the Green and Gurson models to kinematic hardening, Mech. Mater., 35, 1-18.

Brocks W. and Steglich D. (2003). Damage models for cyclic plasticity, in: Key Engineering Materials, vols. 251-252, pp. 389-398.

Devaux J., Gologanu M., Leblond J.B. and Perrin G. (1997). On continued void growth in ductile metals subjected to cyclic loadings, in: Proceedings of the IUTAM Symposium on Nonlinear Analysis of Fracture, Cambridge, GB, J. Willis, ed., Kluwer, pp. 299-310.

Enakoutsa K., Leblond J.B. and Perrin G. (2007). Numerical implementation and assessment of a phenomenological nonlocal model of ductile rupture, Comput. Meth. Appl. Mech. Engng., 196, 1946-1957.

Gao X., Faleskog J. and Fong Shih C. (1998). Cell model for nonlinear fracture analysis

- II. Fracture-process calibration and verification, Int. J. Fract., 89, 375-398.

Gilles Ph., Jullien B. and Mottet G. (1992). Analysis of cyclic effects on ductile tearing 
strength by a local approach of fracture, in: Advances in Fracture/Damage Models for the Analysis of Engineering Problems, ASME Publication AMD - Vol. 137, pp. 269-284.

Gurson A.L. (1977). Continuum theory of ductile rupture by void nucleation and growth: Part I - Yield criteria and flow rules for porous ductile media, ASME J. Engng. Mater. Technol., 99, 2-15.

Hill R. (1967). The essential structure of constitutive laws for metal composites and polycrystals, J. Mech. Phys. Solids, 15, 79-95.

Kobayashi H., Kusumoto T. and Nakazawa H. (1991). Cyclic J-R-curve and upper limit characteristic of fatigue crack growth in 2-1/2 Cr-Mo steel, 11th International Conference on Structural Mechanics in Reactor Technology (SMIRT 11), Tokyo, Japan, paper $\mathrm{G} 27 / 1$.

Koplik J. and Needleman A. (1988). Void growth and coalescence in porous plastic solids, Int. J. Solids Structures, 24, 835-853.

Leblond J.B. and Morin L. (2014). Gurson's criterion and its derivation revisited, ASME J. Appl. Mech., 81, 051012-1 to 7.

Leblond J.B., Perrin G. and Devaux J. (1995). An improved Gurson-type model for hardenable ductile metals, Eur. J. Mech. A/Solids, 14, 499-527.

Mandel J. (1964). Contribution théorique à l'étude de l'écrouissage et des lois d'écoulement plastique, in: Proceedings of the 11th International Congress on Applied Mechanics, Munich, FRG, Springer, pp. 502-509 (in French).

Mbiakop A., Constantinescu A. and Danas K. (2014). On void shape effects of periodic elasto-plastic materials subjected to cyclic loading, Eur. J. Mech. A/Solids, 49, 481-499.

Rabold F. and Kuna M. (2005). Cell model simulation of void growth in nodular cast iron under cyclic loading, Comput. Mater. Sci., 32, 489-497.

Rice J.R. and Tracey D.M. (1969). On the ductile enlargement of voids in triaxial stress fields, J. Mech. Phys. Solids, 17, 201-217.

Schmidt R.A., Wilkowski G.M. and Mayfield M.E. (1991). The International Piping Research Group (IPIRG) program. An overview, 11th International Conference on Structural Mechanics in Reactor Technology (SMIRT 11), Tokyo, Japan, paper G23/1.

Steglich D., Pirondi A., Bonora N. and Brocks W. (2005). Micromechanical modelling of cyclic plasticity incorporating damage, Int. J. Solids Structures, 42, 337-351.

Tvergaard V. (1981). Influence of voids on shear band instabilities under plane strain conditions, Int. J. Fracture, 17, 389-407. 


\section{HIGHLIGHTS}

- Numerical simulations of porous cells evidence a « ratcheting » of the porosity under cyclic loadings.

- Gurson's model does not reproduce such an effect, due to an oversimplified description of strain hardening.

- An improved variant of this model is proposed and shown to better reproduce the results of the simulations. 